\title{
Transcriptional control of GABAergic neuronal subtype identity in the thalamus
}

\author{
Katherine Sellers ${ }^{1,2}$, Verena Zyka ${ }^{1}$, Andrew G Lumsden ${ }^{1}$ and Alessio Delogu ${ }^{1,2^{*}}$
}

\begin{abstract}
Background: The thalamus is often defined as the 'gateway to consciousness', a feature that is supported by the specific connectivity and electrophysiological properties of its neurons. Inhibitory GABAergic neurons are required for the dynamic gating of information passing through the thalamus. The high degree of heterogeneity among thalamic GABA neurons suggests that, during embryonic development, alternative differentiation programmes exist to guide the acquisition of inhibitory neuron subtype identity.

Results: Taking advantage of the accessibility of the developing chick embryo, we have used in ovo manipulations of gene expression to test the role of candidate transcription factors in controlling GABAergic neuronal subtype identity in the developing thalamus.

Conclusions: In this study, we describe two alternative differentiation programmes for GABAergic neurogenesis in the thalamus and identify Helt and Dlx2 as key transcription factors that are sufficient to direct neuronal progenitors along a specific differentiation pathway at the expense of alternative lineage choices. Furthermore, we identify Calb2, a gene encoding for the GABA subtype marker calretinin as a target of the transcription factor Sox14. This work is a step forward in our understanding of how GABA neuron diversity in the thalamus is achieved during development and will help future investigation of the molecular mechanisms that lead up to the acquisition of different synaptic targets and electrophysiological features of mature thalamic inhibitory neurons.
\end{abstract}

Keywords: Helt, DIx2, Sox14, GABAergic thalamus, Neurogenesis, Diencephalon

\section{Background}

The thalamus plays a crucial function in ensuring faithful transfer of sensory information to the cortex. Excitatory, glutamatergic relay neurons constitute the largest neuronal type in the thalamus. However, thalamic function is not restricted simply to relaying information to and from the cortex: the thalamus can highlight certain inputs and suppress others, a feature that is evident during deep sleep when coordinated oscillations in the thalamo-cortical system suppress the ascending flow of peripheral and sensory inputs [1], or in pathological conditions such as schizophrenia [2] and absence epilepsy [3] when, respectively, hallucinations and temporary loss of consciousness can occur. The ability to modulate the flow of information is supported by a second abundant neuronal type, the

\footnotetext{
* Correspondence: alessio.delogu@kcl.ac.uk

'MRC Centre for Developmental Neurobiology, King's College London, London SE1 1UL, UK

2Present address: Department of Neuroscience, Institute of Psychiatry, King's College London, London SE5 9NU, UK
}

(c) 2014 Sellers et al.; licensee BioMed Central Ltd. This is an Open Access article distributed under the terms of the Creative Commons Attribution License (http://creativecommons.org/licenses/by/2.0), which permits unrestricted use, distribution, and reproduction in any medium, provided the original work is properly credited. The Creative Commons Public Domain Dedication waiver (http://creativecommons.org/publicdomain/zero/1.0/) applies to the data made available in this article, unless otherwise stated. inhibitory GABA neurons of the reticular nucleus of the thalamus (TRN). TRN neurons are defined by the expression of the parvalbumin gene [4], their fast spiking action and specific connectivity, which is strictly confined within the thalamus. While TRN neurons are a near homogeneous population, the remaining GABA inhibitory neurons in the thalamus (nonTRN GABA thalamus) differ from those of the TRN in their anatomical position, morphology, connectivity, and molecular profile, and consequently also function. NonTRN GABA neurons can be grouped into two large categories: local interneurons active within the thalamus and projecting neurons with extra-thalamic targets. The TRN neurons derive from GABAergic progenitors in the embryonic prethalamic (pTh) compartment [5]. Whilst it was long thought that all thalamic inhibitory neurons originate in the pTh, it is now clear that the thalamus proper contains a resident population of neuronal progenitors fated to become inhibitory. Most nonTRN GABA neurons develop from a progenitor type in the rostral part of the thalamus (rTh) $[6,7]$. 


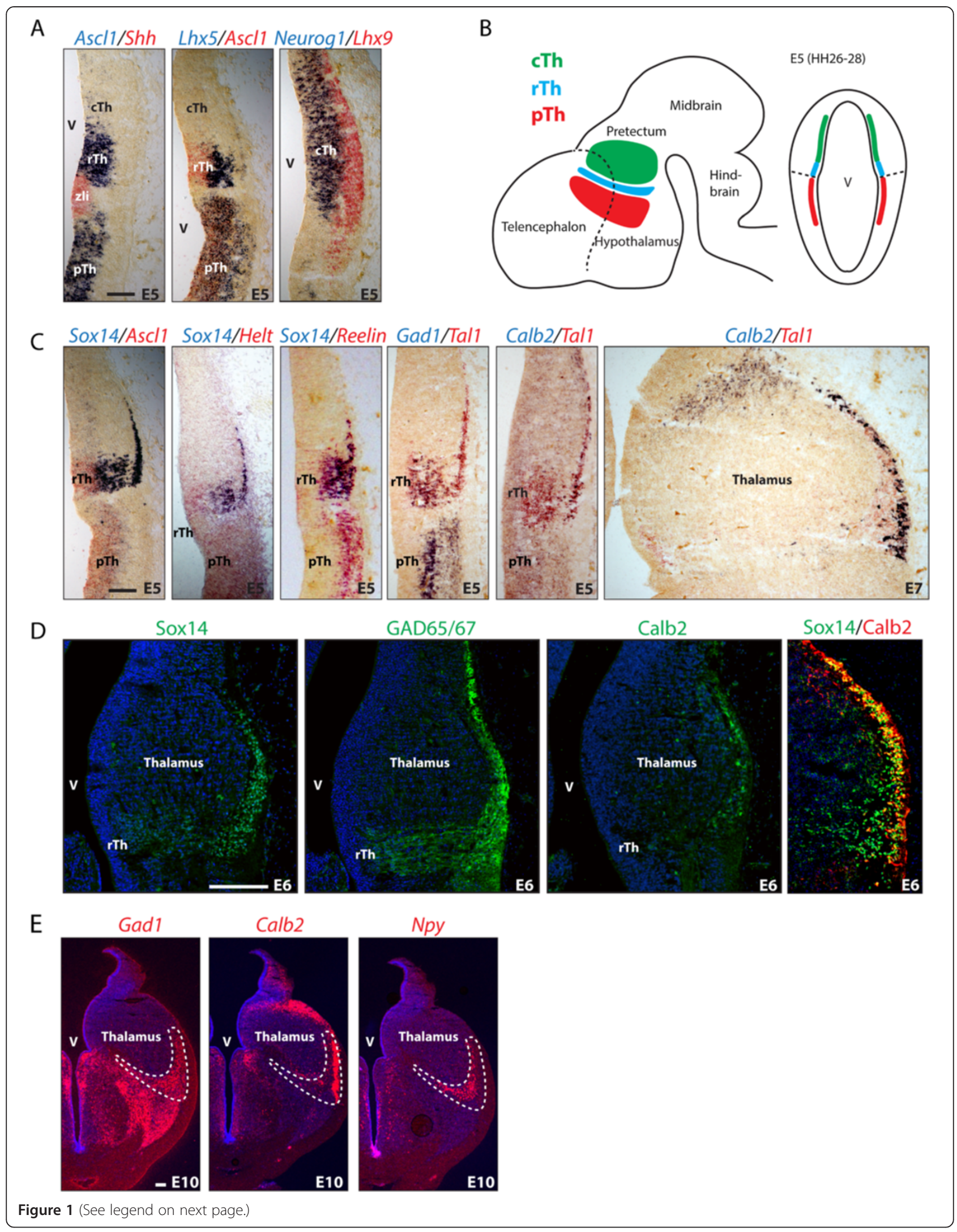


(See figure on previous page.)

Figure $1 \mathrm{GABA}$ progenitor domains in the chick thalamus. Progenitor territories with a GABAergic fate in the thalamus and pTh are visualized by expression of $A s C l 1$ and $L h \times 5$ and are present on both sides of the regional organizer zli, labeled by Shh expression. Thalamic progenitors positioned further away from the zli acquire a glutamatergic fate and express Neurogl and $L h \times 9$ (A). Schematic drawing illustrating the position of prethalamic (pTh), rostral thalamic (rTh) and caudal thalamic (cTh) progenitor domains in lateral and coronal views of the developing chick brain (B). Helt, Tal1 and Sox14 are expressed in rTh progenitors and precursors. Their expression overlaps with GABAergic markers Gad1 and Reelin. From the fifth day of incubation (E5), rTh GABA derivatives express the interneuron subtype marker and calcium binding protein Calretinin (Calb2) (C). E6 rTh neurons can be visualized by immunodetection of the GABA-synthesizing enzymes GAD65/67. The calcium binding protein Calb2 is present and co-expressed with the transcription factor Sox14 in a subset of rTh neurons (D). Position of rTh progenitors in the perirotundic area of the chick thalamus by day E10 of embryonic development. The Calb2-expressing neurons appear to segregate from Npy-expressing neurons within the perirotundic area and may represent two subtypes within the rTh pool. Both cell groups are labeled by the panGABAergic marker Gad1 (E). Scale bars: $100 \mu \mathrm{m}$.

Acquisition of cell lineage identity in the developing thalamus is regulated by the activity of a local organizer, the zona limitans intrathalamica (zli) acting via secretion of the morphogen molecules Shh, Wnts and FGFs [8-12]. Our and other groups have shown that different developmentally regulated transcription factors are induced by zli signaling on both sides of the organizer [8-10,12-17]. We have recently reported that in the pTh the pro-GABAergic transcription factors Dlx1/2, which are required for development of the TRN [5], suppress the rTh nonTRN GABA differentiation programme [6]. This finding is consistent with the recent discovery that the rTh Gata2 gene exerts a similar and reciprocal function, by suppressing pTh TRN fate [18]. Both the $D l x 1 / 2$ and Gata2/3 loss-of-function data suggest that on each side of the diencephalic organizer zli, two alternative developmental programmes lead to the formation of GABAergic neurons. Here, we have investigated further some of the transcriptional events that define the differentiation trajectories of the two main subtypes of thalamic GABAergic neurons using the chick embryo system. Firstly, we demonstrate that the transcription factor Helt is sufficient to drive differentiation along the nonTRN GABA programme within the thalamus. Second, we confirm and provide novel evidence that Dlx2 acts as a lineage specification factor within the pTh to induce TRN fate at the expenses of the nonTRN one. Third, we report that Sox14, a post mitotic transcription factor expressed in the rTh, is necessary and sufficient to confer some nonTRN GABA neuron subtype features. In conclusion, this work provides new experimental evidence towards understanding the transcriptional programmes driving the acquisition of GABAergic subtype diversity in the thalamus.

\section{Results and discussion}

Asymmetric GABAergic neurogenesis at the Th/pTh border Similar to the mouse, the chick thalamus expresses the panGABAergic marker Gad1 in the pTh and rTh (Figure 1C). Mirroring Gad1 expression are GABArelated transcription factors Ascl1 and $L h x 5$ (Figure 1A). Both progenitor domains, the $\mathrm{pTh}$ and $\mathrm{rTh}$, also express
Reelin (Figure 1C). We recently defined the $\mathrm{rTh}$ in the mouse by the sequential expression of Helt, Tal1 and Sox14 transcription factor genes [6]; a similar cascade of homologous genes defines the rTh avian equivalent (Figure 1C). In the chick, post mitotic rTh neurons express the GABA subtype marker Calb2 (Figure 1C, D, E) and, in common with the mouse, the Npy GABA subtype marker. Later in embryonic development, Sox14 positive rTh derivatives appear to segregate in a lateral Calb2 positive domain and a medial Npy positive domain (Figure 1E). The larger progenitor domain in the caudal thalamus (cTh) (Figure 1B) expresses Neurog2 and $L h x 9$ (Figure 1A) and acquires a glutamatergic fate, as described already in other model systems: the zebrafish [14] and the mouse [7].

\section{Helt is sufficient to induce rostral thalamic identity in the thalamus}

While gain- and loss-of-function experiments have revealed an important function for Helt in the embryonic mouse midbrain $[19,20]$; Helt's role in the developing diencephalon remains more ambiguous. Helt is required for GABAergic differentiation in the pretectum, but not in the rTh [6] and ectopic expression of the gene was not sufficient to induce the GABA fate in the mouse diencephalon, possibly due to the reported early lethality of the transgenic strategy used [21]. We took advantage of the accessibility of the chick embryo to perform in ovo electroporation of a constitutively active Helt expression construct in the diencephalon. Ectopic Helt expression in the thalamus prior to the onset of neurogenesis (E2.5) suppresses the glutamatergic fate, indicated by the lack of Neurog2 and $L h x 9$ expression by E5 (Figure 2A) and concomitant differentiation towards a rTh subtype that expresses the panGABA markers Ascl1, Gad1 and Reelin and rTh transcription factor genes Gata2, Tal1, Sox14 (Figure 2B, C). Taken together, these data led us to conclude that ectopic expression of Helt in the cTh progenitor domain is sufficient to divert the fate of these progenitors from glutamatergic to GABAergic and more specifically towards a rTh GABAergic fate (Figure 2E). It should be noted that all rTh markers analyzed are also 


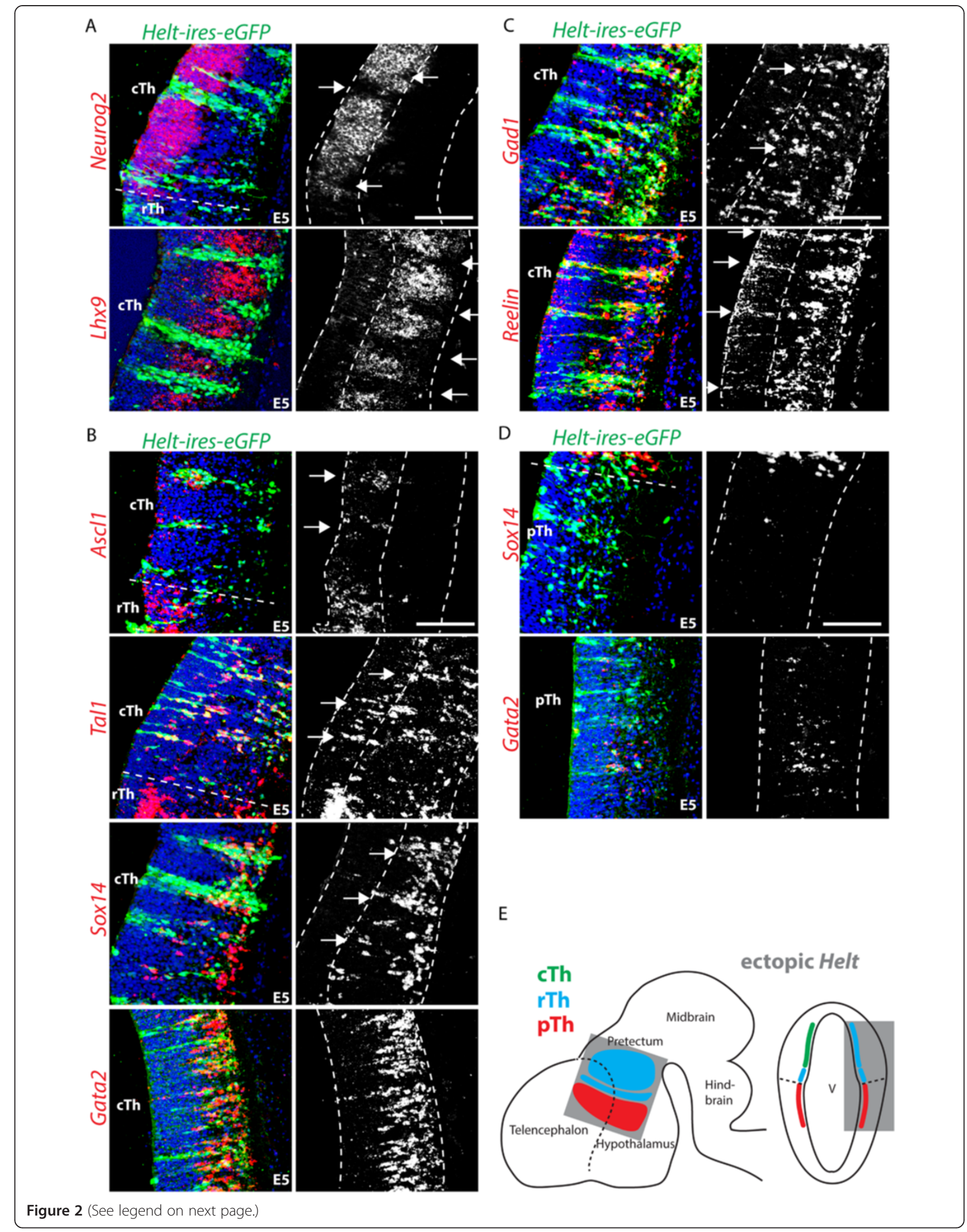


(See figure on previous page.)

Figure 2 Helt expression is sufficient for rostral thalamic (rTh) subtype specification. Ectopic expression of the rTh transcription factor Helt in the caudal thalamic (CTh) glutamatergic progenitor domain at E2.5, suppresses Neurog2 and Lhx9 expression, indicative of a loss of glutamatergic progenitor identity (A). CTh progenitors expressing ectopic Helt, acquire expression of all rTh markers tested: proliferative zone (Ascl1) and postmitotic compartment (Gata2, Tal1 and Sox 14) (B). PanGABAergic markers are also induced (Gad1 and Reelin) (C). Ectopic Helt expression in the prethalamic (pTh) GABA progenitor domain fails to induce rTh subtype identity, suggesting that other co-factors may be required (D). Schematic drawing summarizing the effect of ectopic Helt expression in the three progenitor domains of the thalamus: CTh, rTh and pTh (E). Scale bars: $100 \mu \mathrm{m}$.

expressed by some GABAergic neurons in other brain compartments (for example, the caudal pretectum and the ventral and dorsal midbrain); it is, therefore, solely on the grounds that electroporated cells expressing Helt ectopically are contained within the thalamus that we conclude they have acquired a rTh identity.

pTh and thalamic domains are separated by the zli, a morphological and molecular landmark. We used expression of Shh, visualized by in situ hybridisation (ISH) on consecutive coronal brain sections (data not shown) to draw a dotted line dividing $\mathrm{pTh}$ and thalamic compartments (Figure 2A, B, D). In contrast to the effect seen in the thalamus, ectopic expression of Helt in the pTh failed to induce markers of rTh subtype identity (Figure 2D), implying that the activity of prepatterning genes, such as Irx3 $[8,13]$ may be required for the observed thalamic phenotype.

\section{DIx2 induces prethalamic GABAergic neurogenesis in the thalamus}

Expression of $D l x 2$ defines neuronal progenitors in the pTh and is not expressed in the rTh. Dlx2 regulates GABA progenitor migration and differentiation in the telencephalon [22] and the prethalamus [5] but its role in lineage fate decisions in the diencephalon has received limited attention [23,24]. We have shown that in the absence of both $D l x 1$ and $D l x 2$ genes ( $D l x 1 / 2$ compound knockout), the mouse pTh acquires a rTh fate, leading to the formation of an ectopic intergeniculate leaflet (IGL) expressing Npy [6]. We also observed how the ectopic rTh that forms in the pTh of Dlx1/2 compound mutant mice lacks expression of the earliest rTh marker Helt. This is supportive of a model whereby GABA progenitors retain latent plasticity for subtype identity upon cell cycle exit. The model finds further support in the recent report that Gata2 and Gata3 act post mitotically in the rTh to suppress the alternative $\mathrm{pTh}$ fate by suppressing $D l \times 2$ expression [18]. To test whether the reciprocal regulation is also true, we investigated Gata2 and Gata3 expression in the pTh of the $D l x 1 / 2$ compound knockout mouse (Figure 3A). In agreement with our previous observation [6] the Gata2 and Gata3 genes are also ectopically expressed in the pTh of $D l \times 1 / 2$ mice, further confirming that Dlx1/2 act as lineage identity factors to support a pTh GABA subtype fate. To further investigate the role of Dlx2 in cell lineage decisions, we forced expression of
Dlx2 in the thalamus. Forced expression of Dlx2 in the cTh induces a glutamatergic to GABAergic fate switch as shown by the induction of the proneural gene Ascl1 and panGABA marker Gad1 (Figure 3B, E); ectopic GABA differentiation driven by Dlx2 presents features of pTh identity (Arx, Meis2) (Figure 3C); this result is consistent with the previous observation of ectopic induction of the $D l \times 1 /$ 2 effector gene $A r x$ under similar overexpression conditions [5]. Ectopic $D l x 2$ expression in the rTh does not alter its overall GABAergic fate, but suppresses rTh GABA subtype identity (Tal1, Sox14) (Figure 3B, D, E).

\section{Sox14 is required for rostral thalamic subtype marker expression}

Sox14 is expressed by GABAergic progenitors in the rTh upon cell cycle exit [6]. We described a Sox14 loss of function phenotype in the developing mouse diencephalon, suggestive of a role in positioning of rTh derivatives in the developing thalamus [6]. A possible role for Sox14 in regulating some aspects of subtype identity is supported by our previous observation that the proportion of $\mathrm{rTh}$ neurons expressing the GABA subtype marker $N p y$ is increased in the embryonic Sox14 knockout mouse, a phenotype that, we speculate, results from retention of ventral IGL derivatives within the presumptive IGL. Here, we have adopted a chick-specific RNA interference strategy, based on the U6 promoter expression of a microRNA operon [25], to achieved acute Sox14 knockdown (Sox14i) in the developmental time window between E2.5 and E5.5 (Figure 4A). rTh progenitors with reduced Sox14 expression retain their GABAergic fate, as indicated by the presence of the GABA enzymes Gad65/67 and expression of the rTh genes Ascl1, Tal1 and Npy (Figure 4B), but fail to express the GABA subtype marker Calb2 (Figure 4C).

To investigate further the relationship between Sox14 and Calb2, we investigated the effects of ectopic expression of Sox14 in the pTh Calb2 negative GABAergic domain. Consistent with the observed loss of Calb2 expression in the Sox14i rTh, we observed a rapid upregulation of Calb2 in pTh GABA progenitors upon ectopic Sox14 expression (Figure 5A). Ectopic Calb2 protein can be detected in the more mature neurons expressing ectopic Sox14 but is absent in proliferating progenitors (Figure 5B), the delayed onset of expression is reminiscent of the late onset of Calb2 expression seen in rTh-derived neurons (Figure 1C, D). Gata2, a rTh marker active 


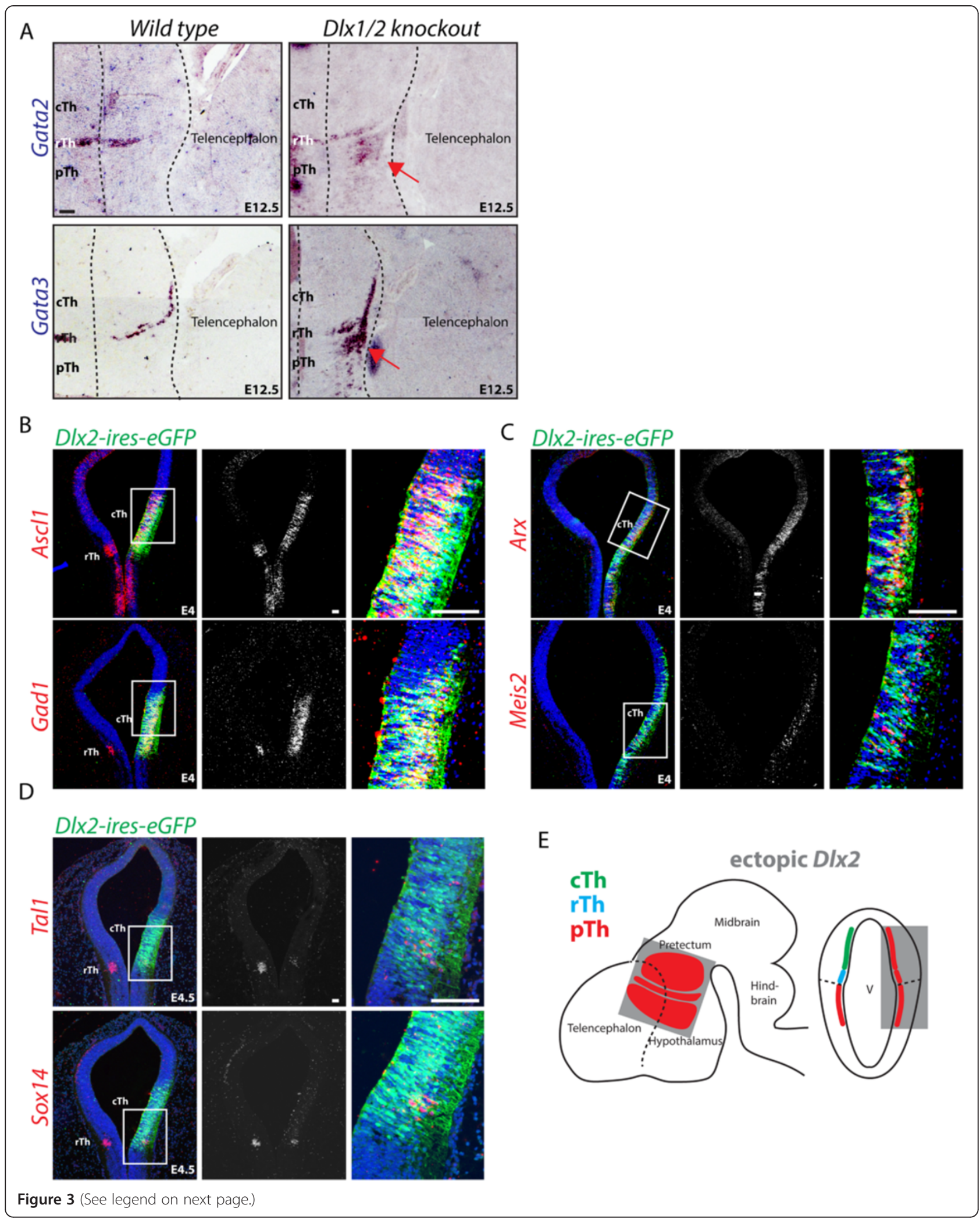


(See figure on previous page.)

Figure 3 Dlx2 induces GABAergic differentiation and suppresses rostral thalamic (rTh) subtype identity. Key rTh transcription factor genes Gata2 and Gata3 are expressed in the pTh of D/x1/2 compound knockout mice (red arrows), providing strong evidence that D/x1/2 are required to maintain pTh identity (A). Ectopic expression of the pTh gene D/x2 in the glutamatergic CTh, induces GABAergic differentiation, visualized by induction of the panGABAergic markers Gad1 and proneural gene Ascl1 (B). Ectopic expression of D/x2 induces pTh GABA subtype progenitor markers in the rTh and CTh (Arx, Meis2) (C). Consistent with a role in controlling GABA subtype identity in the thalamus, rTh markers (Tal1, Sox14) are downregulated upon ectopic expression of D/x2 (D). A schematic summary of the effect of ectopic D/x2 expression in the progenitor domains rTh and CTh (E). Scale bars: $100 \mu \mathrm{m}$.

upstream of Sox14 [18] was not induced by ectopic Sox14 (Figure 5A). Similarly, only a minor effect was noticed on the panGABA proneural gene Ascl1 (Figure 5D), possibly due to the predicted function of Sox14 in promoting cell cycle exit [26]. Yet, pTh genes Dlx2 and Meis2 were heavily downregulated upon Sox14 expression (Figure 5C, E). Ectopic Sox14 expression does not interfere with glutamatergic versus GABAergic fate determination in the cTh (not shown), nor does it interfere with general GABA differentiation (Figure 5D, E).

\section{Conclusions}

In this article we describe the organization of neuronal progenitor domains in the chick thalamus on the basis of previous findings in the zebrafish and mouse and conclude that this is well conserved between the three model systems. Taking advantage of the conservation between early neurogenesis in the chick and mouse, we then used in ovo manipulations of gene expression to further investigate the presence of two alternative transcriptional programmes on both side of the middiencephalic organizer zli that guide neuronal progenitor differentiation down the GABAergic pathway, thus generating distinct thalamic inhibitory subtypes. The accessibility of the chick embryo and its amenability to in vivo manipulations facilitated the discovery of novel functions for three developmentally regulated transcription factors: Helt, Dlx2 and Sox14, which contribute to specify GABAergic neuron diversity in the thalamus. Helt is sufficient to divert cTh progenitors towards a rTh GABA subtype fate. This function is likely to depend on the previous expression of prepatterning genes that confer thalamic identity over the pTh one, as Helt expression in the pTh does not change GABA subtype identity. In agreement with previous observations [5], we confirm that Dlx2 is sufficient to induce the GABAergic fate in excitatory thalamic progenitors, inducing expression of Ascl1, Gad1, Meis2 and Arx. Furthermore Dlx2 can suppress the rTh GABA subtype fate via downregulation of Tal1 and Sox14. Sox14 acts post mitotically to induce the rTh subtype marker and calcium binding protein calretinin (Calb2). Calb2 induction by Sox14 is rapid, as it anticipates the time of normal expression in the rTh (E5.5), but follows nonetheless the stepwise progression along cellular differentiation as it is transcribed in Sox14-expressing pTh neurons in the mantel layer and not in those in the proliferative zone. At the same time, Sox 14 can repress other transcriptional regulators in the pTh, such as Dlx2, Meis 2 and Arx. These new findings add support to the emerging view that differentiating GABAergic neurons retain subtype lineage plasticity upon leaving the cell cycle: in the pTh, Dlx $1 / 2$ suppress a latent $r T h$ fate [[6] and this manuscript], whilst in the rTh, Gata2/3 suppress a latent pTh fate [18]. In both cases, cells have already progressed from progenitors to precursors. Given that a similar transcription factor cascade to the one described for the rTh occurs also at other brain locations, it would seem reasonable to speculate that preexisting patterning genes and extrinsic factors play an essential role in shaping up the full transcriptional profile of otherwise similar GABAergic neurons in functionally distinct anatomical regions. The evolution of two alternative and mutually exclusive GABAergic differentiation programmes in the thalamus may be instrumental to achieve a broad spectrum of inhibitory synaptic connectivity and electrophysiological properties at this crucial anatomical node along corticopetal and corticofugal pathways. It should be considered that, whilst the organization of the main neuronal progenitor domains is conserved, different cell sorting and migratory pathways shape the adult thalamus in the different model systems, hence the main adult structure derived from rTh progenitors is the IGL in the mouse and the perirotundic area in the avian system [27,28]. Whilst we have further characterized the developing IGL as a source of tangentially migrating GABA neurons in the thalamus [6], it remains unknown whether the avian perirotundic area asserts a similar function. In consideration of the broad functional heterogeneity of GABA neurons in the thalamus, it is likely that transcriptional regulation continues to play a crucial role well after the initial differentiation of $\mathrm{rTh}$ and $\mathrm{pTh}$ GABA neurons, to further guide the acquisition of diverse cellular properties.

\section{Methods}

\section{Chick and mouse embryos}

Fertilized hens eggs (Henry Stewart, Louth, UK) were incubated in a humidified room at $38^{\circ} \mathrm{C}$ for 3 to 6 days before harvesting and dissecting in cold PBS for whole 


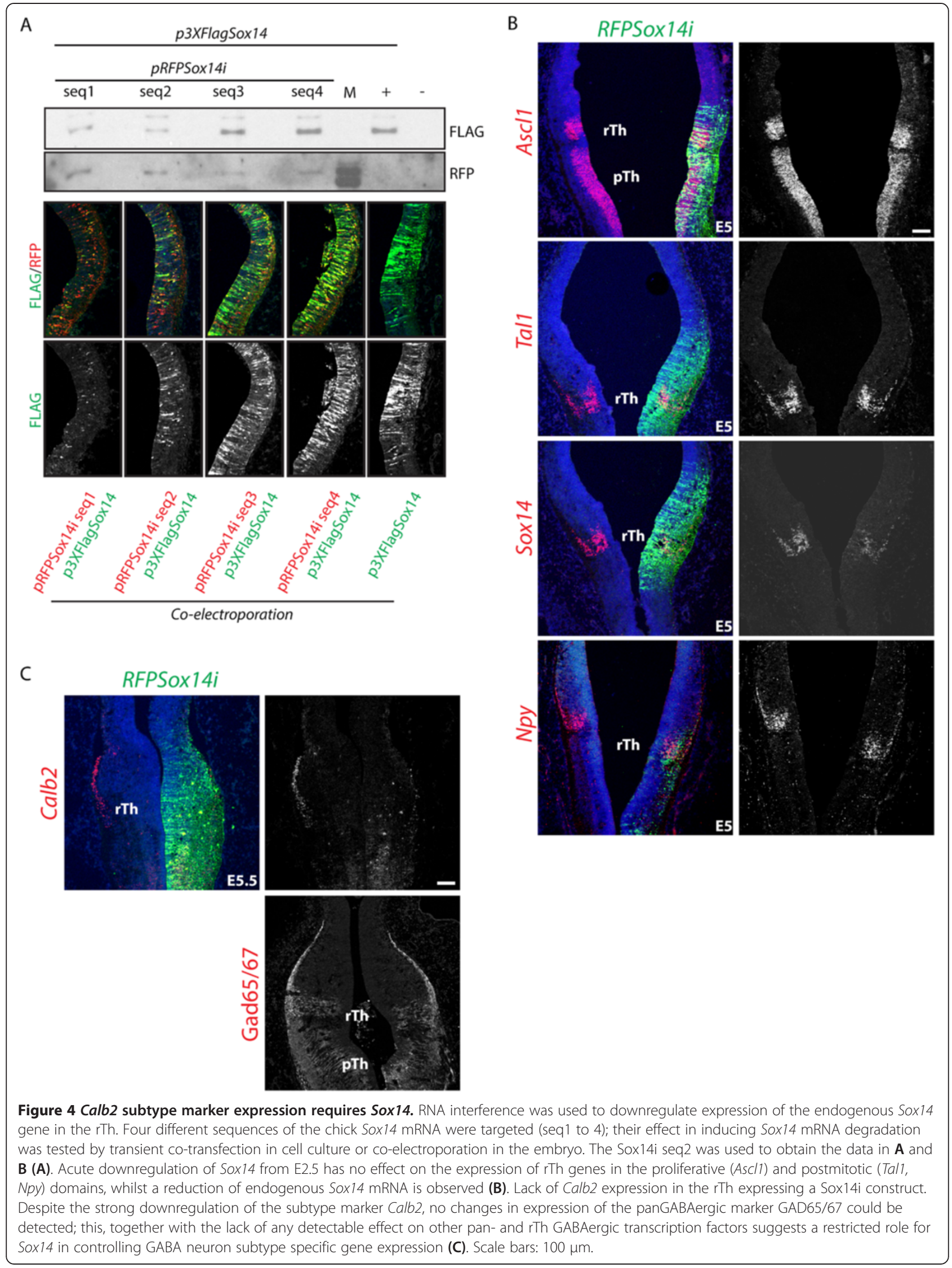



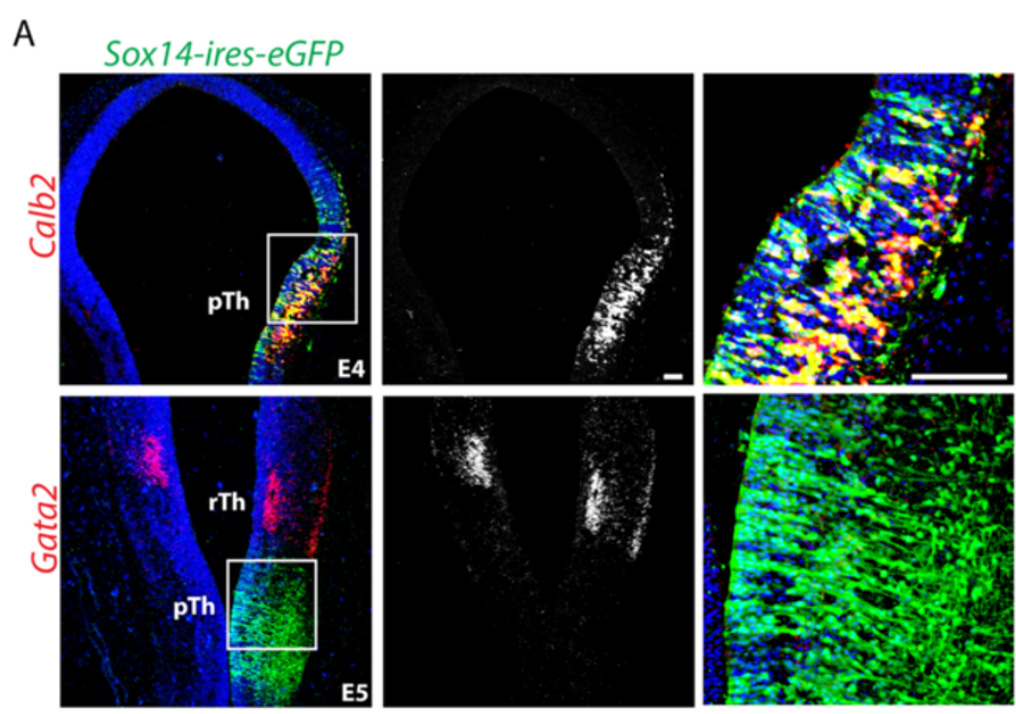

C

Sox14-ires-eGFP
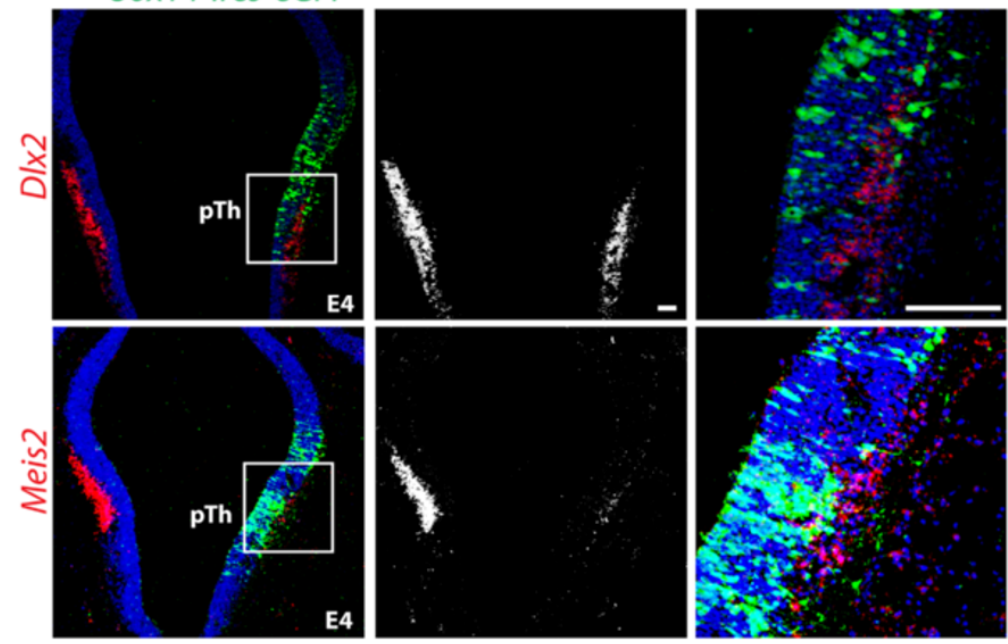

D Sox14-ires-eGFP
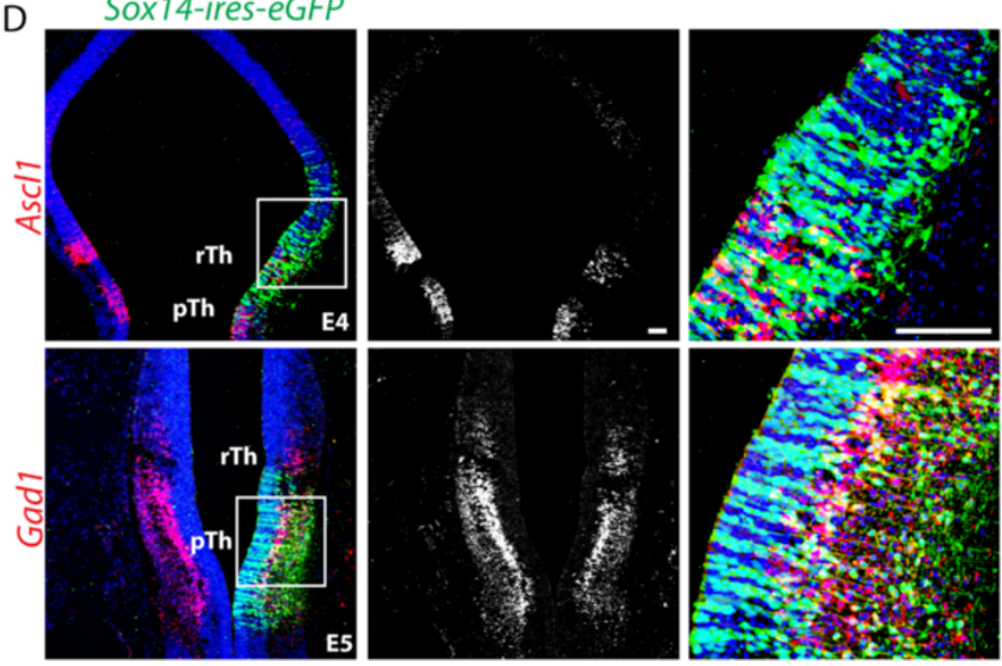

Figure 5 (See legend on next page.)

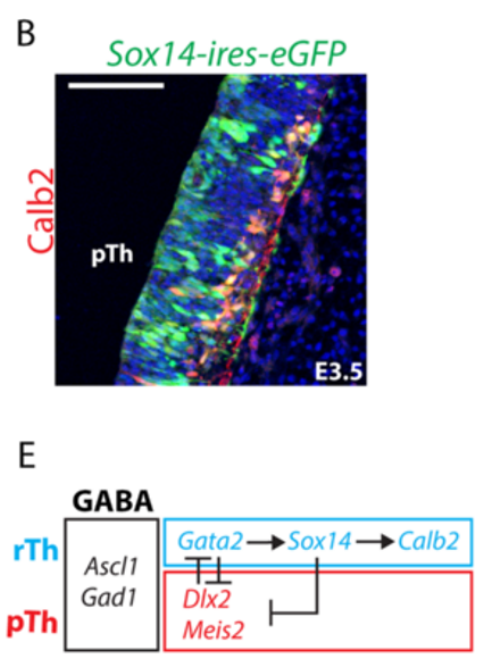


(See figure on previous page.)

Figure 5 Sox14 expression induces Calb2 and suppresses prethalamic (pTh) GABA identity. Sox14 expression in pTh GABA progenitors results in the selective activation of the rTh subtype marker Calb2, whilst the upstream regulator of rTh identity Gata2 was not activated (A). Immunohistochemistry illustrating the co-localization of Calb2 protein in pTh progenitors expressing the transcription factor Sox 14 (B). Expression of pTh progenitor markers (D/x2 and Meis2) is reduced upon ectopic expression of Sox 14 (C), while the overall GABAergic identity is not affected (Gad1) (D). Schematic recapitulation of the cross-regulation of GABA subtype restricted transcription factor on the rostral and pTh sides of the zli. Gata2/3 and DIx1/2 have similar but reciprocal roles in suppressing the alternative subtype fate. Sox14, which acts downstream of Gata2 controls transcription of a subtype marker gene: Calb2. Its ectopic expression is not compatible with that of pTh transcription factor genes (E). Scale bars: $100 \mu \mathrm{m}$.

mount ISH. Staging of chicken embryos was according to the embryonic day (E) since egg incubation began. Fixed (4\% PFA) double knockout $D l \times 1 / 2$ mouse embryos and littermate controls were a kind gift from John L Rubenstein (UCSF). Mice were bred and maintained at UCSF under local ethical and legal regulations.

\section{Gain and loss of function DNA vectors}

To achieve a knockdown effect on the expression level of endogenous Sox14, we adopted the strategy described in [25]. Briefly, 22 nucleotide target sequences were identified using an siRNA design tool available at (http://sirna. wi.mit.edu) (Whitehead Institute for Biomedical Research) starting at positions: 299, 500, 545 and 745 of NCBI sequence NM_204761. Sox14 specific oligonucleotides and a scrambled sequence were subsequently cloned in the second miRNA site of vector pRFPRNAi to generate synthetic miRNA30-like hairpins under the control of the chick U6 snRNA promoter, as described in [25].

To drive ectopic expression of Helt, a mouse cDNA containing the entire coding sequence (CDS) of the gene was cloned downstream of the constitutive promoter of the pCAGGS vector to transcribe a bicistronic mRNA containing ires-eGFP. Similarly, to drive expression of Sox14, the entire CDS of the chick gene was cloned in the pCAGGS-ires-eGFP vector. The CDS of chick Sox14 was also cloned in frame with three FLAG tags in the p3XFLAG-CMV-7.1 (Sigma-Aldrich, St.Louis, Missuouri, USA) to generate an N-terminus FLAG-tag version of the protein. Ectopic expression of mouse $D l x 2$ was obtained using a constitutive expression vector (pCAGGS) kindly provided by John L Rubenstein.

\section{Evaluation of gene knockdown efficiency}

In order to assess which of the four pRFPSox14i plasmids had the strongest knockdown effect, each pRFPSox14i DNA and an equimolar amount of p3XFLAGSox14 was co-transfected in cultured COS cells using a standard Lipofectamine protocol (Thermo Fisher Scientific, Massachusetts, USA). Total cell lysate for each Sox $14 i$ combination was diluted 400 times and used as substrate to detect FLAG and RFP proteins by Western blotting (mouse anti-FLAG Sigma F1804; used at a dilution of 1:3,000) and rabbit anti-RFP (Millipore, Massachusetts,
USA AB3216; used at a dilution of 1:500). To test for downregulation in vivo, each pRFPSox14i vector in equimolar combination with the p3XFLAGSox14 was coelectroporated in the diencephalon of E2.5 chick embryos. Electroporated embryos were collected after 48 hours of incubation and processed for immunohistochemical detection with mouse anti-FLAG (Sigma F1804; used at a dilution of 1:1,000) and rabbit anti-RFP (Millipore, Massachusetts, USA AB3216; used at a dilution of 1:100) on coronal sections.

\section{In ovo electroporation}

Chick embyros were electroporated at E2.5 using fine platinum electrodes and an ElectroSquare Porator, Harvard Apparatus, Massachusetts, USA. Settings were as follows; $15 \mathrm{~V}, 3$ pulses, $50 \mathrm{~ms}$ pulse duration, $950 \mathrm{~ms}$ interval. DNA was diluted to a final concentration of $1 \mu \mathrm{g} / \mathrm{ml}$ and fast green added to aid visualization. DNA injected into the neural tube at diencephalon level; electrodes were placed either side of the diencephalon over the thalamus or prethalamus. DNA overexpression vectors plasmids used; pCAGGS-Helt-ires-eGFP, pCAGGS-Sox14ires-eGFP, pCAGGS-Dlx2 (gift from John L Rubenstein, UCSF) (co-electroporated with pCAGGS-eGFP), pRFPSox 14i(1-4), pRFPscrambled and p3XFLAGSox14.

\section{In situ hybridisation and immunohistochemistry}

Electroporated and control embryos were dissected in ice-cold PBS and cryoprotected by passage through serial dilutions of sucrose/PBS equilibration; 10\%, 20\% and finally $30 \%$ sucrose/PBS. Samples were then embedded (Tissue Tek, Sakura, Torrance, Canada) and frozen by floating on liquid nitrogen. Cryosections were cut at 12$\mu \mathrm{m}$ thickness. ISH was carried out as published in [29]. In brief: hybridisation with digoxigenin (DIG) and or fluorescein isothiocyanate (FITC; Roche, Basel, Switzerland) labeled riboprobes was carried out overnight at $65^{\circ} \mathrm{C}$. Washing steps were carried out in MABT (100 mM maleic acid, $150 \mathrm{mM} \mathrm{NaCl}, 0.1 \%$ Tween 20). Blocking was performed in 2\% Boehringer Blocking Reagent (BBR) (Roche, Basel, Switzerland), 20\% normal goat serum in MABT. The anti-DIG or anti-FITC alkaline phosphatase (AP)-conjugated antibody (Roche, Basel, Switzerland) was incubated overnight at $4^{\circ} \mathrm{C}$. Color reaction was developed 
using NBT/BCIP substrate in NTMT (pH 9.5) or Fast Red in Tris ( $\mathrm{pH}$ 8.2). For two color ISH, the first APconjugated antibody was removed from the section via a 30 -minute incubation in $5 \mathrm{mM}$ EDTA/PBS at $70^{\circ} \mathrm{C}$. Chick anti-Gata2 ISH probe was a gift from Anthony Graham (KCL). Mouse anti-Gata2/3 ISH probes were a kind gift from Maxim Bouchard (McGill University). After completion of ISH protocol, anti-GFP immunohistochemistry was carried out in order to identify electroporated cells. Sections were blocked in 5\% normal goat serum with $0.25 \%$ Triton X-100 for one hour at room temperature, rabbit anti-GFP (Thermo Fisher Scientific, Massachusetts, USA A11122, 1:100 dilution) added to block incubated overnight at $4^{\circ} \mathrm{C}$, secondary antibody goat anti-rabbit Alexa488 (Thermo Fisher Scientific, Massachusetts, USA; added to block at a dilution of 1:500), incubated for 1 hour at room temperature. Other antibodies used were: guinea pig anti-Sox14 (gift from Thomas Jessell, Columbia University, New York; used at a dilution of 1:5,000), rabbit anti-calretinin (Calb2, AbCam, Massachusetts, USA ab702; used at a dilution of 1:100), rabbit anti-Gad65/67 (AbCam, Massachusetts, USA ab11070; used at a dilution of 1:5,000) rabbit anti-RFP (Millipore, Massachusetts, USA AB3216; used at a dilution of 1:100) and goat secondary Alexa-conjugated antibodies (Thermo Fisher Scientific, Massachusetts, USA; used at a dilution of 1:500). The sections were then nuclear counterstained with $100 \mathrm{ng} / \mathrm{ml}$ Hoechst in PBS for 10 minutes before mounting in antifade Prolong Gold (Thermo Fisher Scientific, Massachusetts, USA). Images were taken with an Eclipse series Nikon, Tokyo, Japan confocal microscope and processed with Image J (rsbweb.nih.gov/ij/).

\section{Abbreviations}

AP: alkaline phosphatase; BBR: Boehringer Blocking Reagent; CDS: coding sequence; cTh: caudal thalamus; DIG: digoxigenin; FITC: fluorescein isothiocyanate; GABA: $\gamma$-aminobutyric acid; IGL: intergeniculate leaflet; ISH: in situ hybridisation; nonTRN: GABAergic neurons in the thalamus excluding the TRN; PBS: phosphate-buffered saline; pTh: prethalamus; rTh: rostral thalamus; TRN: thalamic reticular nucleus; zli: zona limitans intrathalamica.

\section{Competing interests}

The authors declare that they have no competing interests.

\section{Authors' contributions}

$K S, V Z$ and $A D$ carried out experiments and acquired images; $A G L$ and $A D$ provided financial support for the project; $A D, K S$ and $A G L$ designed experiments; $A D$ wrote the manuscript. All authors read and approved the manuscript.

\section{Acknowledgments}

We are grateful to the following people for sharing plasmids for overexpression and/or riboprobe synthesis: M Bouchard, A Graham, J Partanen, J L Rubenstein. We thank J L Rubenstein for sending us fixed D/x1/2 compound knockout embryos. This research was funded by an MRC programme grant to AGL (G0601064) and King's College London (KCL) departmental funding to AD. The MRC programme approved the design of the work and expected data to be collected, analyzed and interpreted. KCL departmental funding sponsored the materials and facilities required for the revision of the manuscript.
Received: 10 January 2014 Accepted: 2 June 2014

Published: 15 June 2014

\section{References}

1. Steriade M: The corticothalamic system in sleep. Front Biosci 2003, 8:d878-d899.

2. Behrendt RP: Dysregulation of thalamic sensory 'transmission' in schizophrenia: neurochemical vulnerability to hallucinations. J Psychopharmacol 2006, 20:356-372.

3. Steriade M: Sleep, epilepsy and thalamic reticular inhibitory neurons. Trends Neurosci 2005, 28:317-324.

4. De Biasi S, Arcelli P, Spreafico R: Parvalbumin immunoreactivity in the thalamus of guinea pig: light and electron microscopic correlation with gamma-aminobutyric acid immunoreactivity. J Comp Neurol 1994, 348:556-569.

5. Cobos I, Broccoli V, Rubenstein JL: The vertebrate ortholog of Aristaless is regulated by Dlx genes in the developing forebrain. J Comp Neurol 2005, 483:292-303.

6. Delogu A, Sellers K, Zagoraiou L, Bocianowska-Zbrog A, Mandal S, Guimera J, Rubenstein JL, Sugden D, Jessell T, Lumsden A: Subcortical visual shell nuclei targeted by ipRGCs develop from a Sox $14^{+}$-GABAergic progenitor and require Sox14 to regulate daily activity rhythms. Neuron 2012, 75:648-662

7. Vue TY, Aaker J, Taniguchi A, Kazemzadeh C, Skidmore JM, Martin DM, Martin JF, Treier M, Nakagawa Y: Characterization of progenitor domains in the developing mouse thalamus. J Comp Neurol 2007, 505:73-91.

8. Kiecker $C$, Lumsden A: Hedgehog signaling from the $Z$ LI regulates diencephalic regional identity. Nat Neurosci 2004, 7:1242-1249.

9. Scholpp $\mathrm{S}$, Wolf $\mathrm{O}$, Brand $\mathrm{M}$, Lumsden A: Hedgehog signalling from the zona limitans intrathalamica orchestrates patterning of the zebrafish diencephalon. Development 2006, 133:855-864.

10. Vue TY, Bluske K, Alishahi A, Yang LL, Koyano-Nakagawa N, Novitch B, Nakagawa Y: Sonic hedgehog signaling controls thalamic progenitor identity and nuclei specification in mice. J Neurosci 2009, 29:4484-4497.

11. Bluske KK, Vue TY, Kawakami Y, Taketo MM, Yoshikawa K, Johnson JE, Nakagawa Y: beta-Catenin signaling specifies progenitor cell identity in parallel with Shh signaling in the developing mammalian thalamus. Development 2012, 139:2692-2702.

12. Kataoka A, Shimogori T: Fgf8 controls regional identity in the developing thalamus. Development 2008, 135:2873-2881.

13. Robertshaw E, Matsumoto K, Lumsden A, Kiecker C: Irx3 and Pax6 establish differential competence for Shh-mediated induction of GABAergic and glutamatergic neurons of the thalamus. Proc Natl Acad Sci U S A 2013, 110:E3919-E3926.

14. Scholpp S, Delogu A, Gilthorpe J, Peukert D, Schindler S, Lumsden A: Her6 regulates the neurogenetic gradient and neuronal identity in the thalamus. Proc Natl Acad Sci U S A 2009, 106:19895-19900.

15. Hashimoto-Torii K, Motoyama J, Hui C-C, Kuroiwa A, Nakafuku M, Shimamura K: Differential activities of Sonic hedgehog mediated by Gli transcription factors define distinct neuronal subtypes in the dorsal thalamus. Mech Dev 2003, 120:1097-1111

16. Kitamura K, Miura H, Yanazawa M, Miyashita T, Kato K: Expression patterns of Brx1 (Rieg gene), Sonic hedgehog, Nkx2.2, Dlx1 and Arx during zona limitans intrathalamica and embryonic ventral lateral geniculate nuclear formation. Mech Dev 1997, 67:83-96.

17. Jeong Y, Dolson DK, Waclaw RR, Matise MP, Sussel L, Campbell K, Kaestner $\mathrm{KH}$, Epstein DJ: Spatial and temporal requirements for sonic hedgehog in the regulation of thalamic interneuron identity. Development 2011, 138:531-541.

18. Virolainen SM, Achim K, Peltopuro P, Salminen M, Partanen J: Transcriptional regulatory mechanisms underlying the GABAergic neuron fate in different diencephalic prosomeres. Development 2012, 139:3795-3805.

19. Nakatani T, Minaki Y, Kumai M, Ono Y: Helt determines GABAergic over glutamatergic neuronal fate by repressing Ngn genes in the developing mesencephalon. Development 2007, 134:2783-2793.

20. Guimera J, Weisenhorn DV, Wurst W: Megane/Heslike is required for normal GABAergic differentiation in the mouse superior colliculus. Development 2006, 133:3847-3857.

21. Miyoshi G, Bessho Y, Yamada S, Kageyama R: Identification of a novel basic helix-loop-helix gene, Heslike, and its role in GABAergic neurogenesis. J Neurosci 2004, 24:3672-3682. 
22. Anderson $\mathrm{SA}$, Eisenstat $\mathrm{DD}$, Shi L, Rubenstein JL: Interneuron migration from basal forebrain to neocortex: dependence on Dlx genes. Science 1997, 278:474-476.

23. Stühmer T, Anderson SA, Ekker M, Rubenstein JLR: Ectopic expression of the Dlx genes induces glutamic acid decarboxylase and Dlx expression. Development 2002, 129:245-252.

24. Long JE, Swan C, Liang WS, Cobos I, Potter GB, Rubenstein JL: Dlx1\&2 and Mash1 transcription factors control striatal patterning and differentiation through parallel and overlapping pathways. J Comp Neurol 2009, 512:556-572.

25. Das RM, Van Hateren NJ, Howell GR, Farrell ER, Bangs FK, Porteous VC, Manning EM, McGrew MJ, Ohyama K, Sacco MA: A robust system for RNA interference in the chicken using a modified microRNA operon. Dev Biol 2006, 294:554-563.

26. Uchikawa M, Kamachi Y, Kondoh H: Two distinct subgroups of Group B Sox genes for transcriptional activators and repressors: their expression during embryonic organogenesis of the chicken. Mech Dev 1999, 84:103-120

27. Yoon MS, Puelles $L$, Redies $C$ : Formation of cadherin-expressing brain nuclei in diencephalic alar plate divisions. J Comp Neurol 2000 427:461-480.

28. Muller K, Hirano S, Puelles L, Redies C: OL-protocadherin expression in the visual system of the chicken embryo. J Comp Neurol 2004, 470:240-255.

29. Strahle U, Blader P, Adam J, Ingham PW: A simple and efficient procedure for non-isotopic in situ hybridization to sectioned material. Trends Genet 1994, 10:75-76.

doi:10.1186/1749-8104-9-14

Cite this article as: Sellers et al:: Transcriptional control of GABAergic neuronal subtype identity in the thalamus. Neural Development 2014 9:14.

\section{Submit your next manuscript to BioMed Central and take full advantage of:}

- Convenient online submission

- Thorough peer review

- No space constraints or color figure charges

- Immediate publication on acceptance

- Inclusion in PubMed, CAS, Scopus and Google Scholar

- Research which is freely available for redistribution 